\title{
Detection of relativistic effects on the S2 orbit with GRAVITY
}

\author{
Marion Grould, Frédéric H. Vincent, Thibaut Paumard and \\ Guy Perrin
}

\author{
LESIA, Observatoire de Paris, PSL Research University, \\ CNRS UMR 8109, Université Pierre et Marie Curie, \\ Université Paris Diderot, \\ 5 place Jules Janssen, 92190 Meudon, France \\ email: marion.grould@obspm.fr \\ email: frederic.vincent@obspm.fr \\ email: thibaut.paumard@obspm.fr \\ email: guy.perrin@obspm.fr
}

\begin{abstract}
The second generation instrument of the VLTI, GRAVITY, is expected to reach an astrometric accuracy of about $10 \mu \mathrm{as}$. It will thus possible to probe the spacetime close to the compact source Sagittarius A* (Sgr A*) at the Galactic Center by using accurate astrometric observations of the second closest star to the Galactic Center, S2. In particular, we show that combining GRAVITY and spectrograph instruments will allow us to detect several relativistic effects such as pericenter advance or the Lense-Thirring effect.
\end{abstract}

Keywords. Galactic Center, Black Hole, General Relativity, S2.

\section{Introduction}

Decades of studies have demonstrated the presence of a compact object of several million solar masses at the center of our Galaxy. Nowadays, the assumption is that this compact object is probably a supermassive black hole described by general relativity. Several ways are investigated in order to confirm the presence of this Kerr black hole such as observing the accretion disk around the central object, the flares near this compact object or the stellar orbits close to Sgr A* (see e.g. Will 2008, Doeleman et al. 2009, Broderick et al. 2011, Vincent et al. 2016 and references therein). This last strategy had in particular permitted to highly constrain the mass of Sgr A* to $4.31 \times 10^{6} \pm 0.42 M_{\odot}$ by using approximatively 15 years of astrometric and spectroscopic measurements of $\mathrm{S}$ stars located in the central parsec (Ghez et al. 2008, Gillessen et al. 2009). In particular, the S2 star corresponding to the second closest star to the Galactic Center and whose orbital period is of about 16 years had mostly contributed to the constraint on this mass.

Several studies have been performed by various authors in order to determine whether relativistic effects could be detected by observing stars orbiting the central compact object (Jaroszynski et al. 1998, Fragile et al. 2000, Weinberg et al. 2005, Zucker et al. 2006, Will 2008, Kannan et al. 2009, Angélil et al. 2010, Bozza et al. 2012, Zhang et al. 2015, $\mathrm{Yu}$ et al. 2016). For instance, Zucker et al. 2006 showed that it is possible to detect the transverse Doppler and gravitational redshifts after 10 years of observations of a dozen of $\mathrm{S}$ stars including the S2 star. This threshold time is evaluated considering observations obtained with astrometric and spectroscopic accuracies of 1.5 mas and $25 \mathrm{~km} / \mathrm{s}$, respectively. Angélil et al. 2010 only considered spectroscopic measurements and estimated the accuracies needed to detect different relativistic effects in only one period of the S2 star: 
$3 \mathrm{~km} / \mathrm{s}$ to detect the effects induced by the Schwarzschild curvature, $10 \mathrm{~m} / \mathrm{s}$ to detect the Lense-Thirring effect or $50 \mathrm{~cm} / \mathrm{s}$ to detect the quadrupole moment of the black hole.

The GRAVITY instrument is expected to observe S stars or closer-in stars with an astrometric accuracy of about $10 \mu$ as. Combining this instrument with a spectrograph is therefore supposed to lead to a detection of different relativistic effects. In particular, we expect that observations obtained on the S2 star will allow constraining both lowand high-order relativistic effects. This is what we will determine in the two following sections.

\section{Detection of different relativistic effects}

In order to predict whether the GRAVITY instrument will be capable of detecting relativistic effects with the S2 star, we developed four stellar-orbit models taking into account a certain number of relativistic effects. These models simulate both astrometric and spectroscopic measurements of the S2 star. The radial velocity data should be obtained by different instruments such as SINFONI at the VLT (Eisenhauer et al. 2003), NIRSPEC at Keck (McLean et al. 1995) or, in the future, MICADO at the E-ELT (Davies et al. 2010). This last spectrograph is expected to reach an accuracy in the order of $1 \mathrm{~km} / \mathrm{s}$ which corresponds to a factor 10 better than the current instruments at VLT and Keck.

Two of our models consider Keplerian orbits and photons propagating in straight line with the velocity of the speed of light $c$. One of these models neglects all relativistic effects, we call this model A. The second model takes into account relativistic effects corresponding to the transverse Doppler redshift and the gravitational redshift, we call this model B. Each of these relativistic redshifts shifts radial velocity of the S2 star of about $100 \mathrm{~km} / \mathrm{s}$ near pericenter passages and less than $10 \mathrm{~km} / \mathrm{s}$ near apocenter passages (Zucker et al. 2006).

The two last models consider the relativistic star trajectory induced by the presence of a Kerr black hole. Thus, they take into account the pericenter advance of the S2 star and the Lense-Thirring effect on the star trajectory (see Fig 1). We also implement in one of these relativistic models the transverse Doppler redshift and the gravitational redshift. These effects are better estimated in this new model than in model B because we take into account the relativistic coordinates of the star to compute these redshifts. This new model does not use ray-tracing code and considers photons propagating in straight line with a velocity $c$ as in models $\mathrm{A}$ and $\mathrm{B}$. All effects induced by the photon curvature are thus neglected in this model such as the gravitational lensing reaching $\approx 20 \mu$ as near S2 pericenter passages. We will call this new model C.

Finally, the last relativistic model, D, is the best model and considers, in addition to the relativistic star trajectory, the relativistic photon trajectory. This model is obtained in full general relativity which means that we use a ray-tracing code to simulate the observations of the S2 star. In particular, we use the GYOTO code developed at the Paris Observatory by Vincent et al. 2011. This model thus accounts for all relativistic effects induced on both star and photon trajectories.

Models $\mathrm{A}$ and $\mathrm{B}$ are described by 8 parameters corresponding to the orbital parameters of the star, the mass and the distance of the black hole. The relativistic models $\mathrm{C}$ and $\mathrm{D}$ are described by three more parameters corresponding to the angular momentum parameters (see legend of Fig 1). They thus depend on 11 parameters.

To determine whether we can detect relativistic effects with $\mathrm{S} 2$ we simulate astrometric and spectroscopic observations of this star with our best model, D. These observations are simulated considering the best fit parameters estimated by Gillessen et al. 2009. The angular momentum parameters of the central black hole are fixed at 

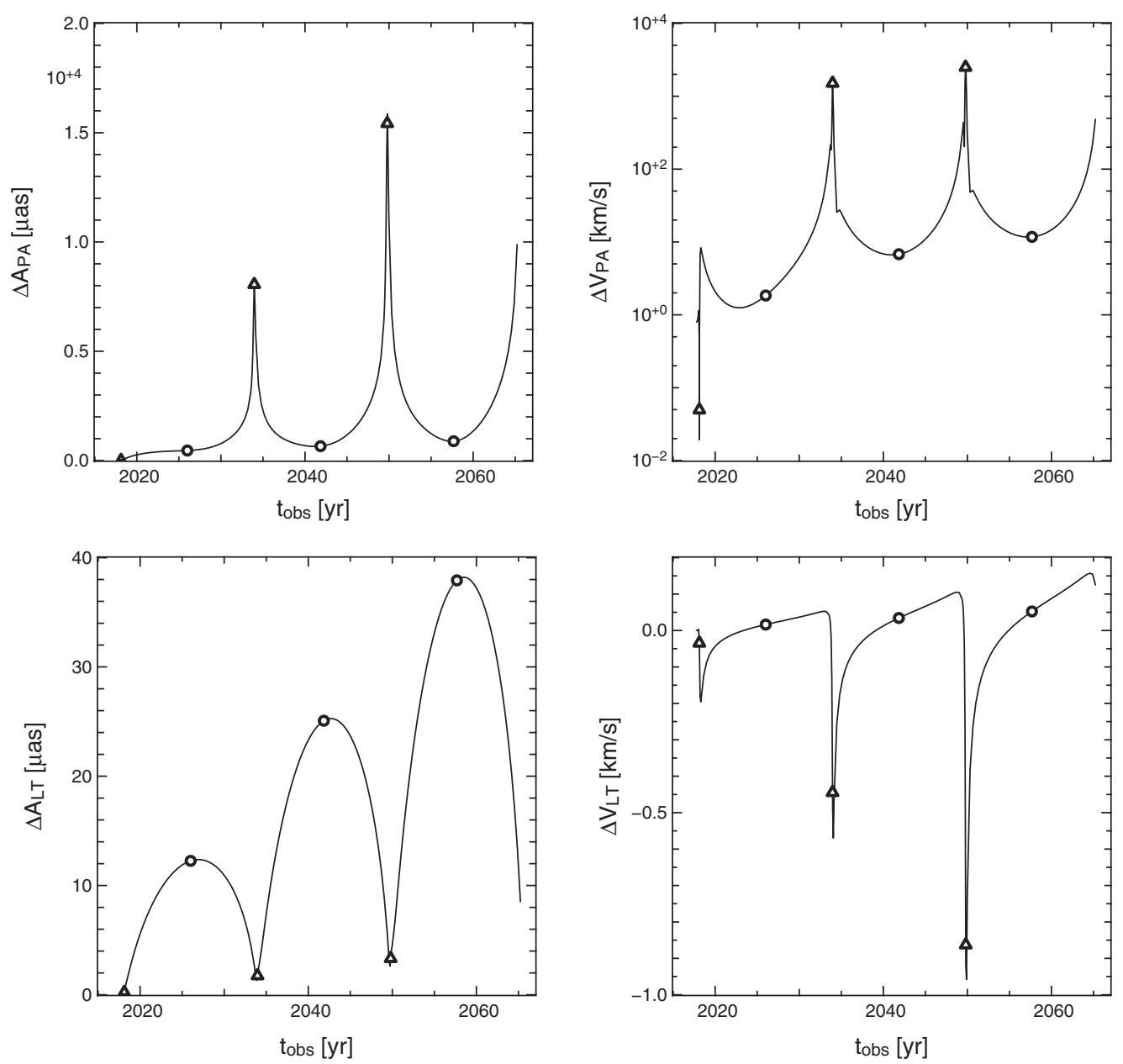

Figure 1. Top: astrometric (left) and spectroscopic (right) impact of the pericenter advance obtained by computing the difference between observations simulated with models B and C. Bottom: astrometric (left) and spectroscopic (right) impact of the Lense-Thirring effect obtained by using model $\mathrm{D}$ and computing the difference between observations generated with $a=0.99$ and $a=0$. The direction of the angular momentum of the black hole is fixed to $\left(i^{\prime}, \Omega^{\prime}\right)=\left(45^{\circ}, 160^{\circ}\right)$ where $i^{\prime}$ corresponds to the angle between the spin-axis and the line of sight of the observer and $\Omega^{\prime}$ corresponds to the angle between the spin-axis projected in the plane of sky and the $\delta$-axis. The open triangles and circles denote the pericenter and apocenter passages, respectively. The different effects are obtained over three full S2 orbital period $(\approx 47$ years $)$.

$\left(a, i^{\prime}, \Omega^{\prime}\right)=\left(0.99,45^{\circ}, 160^{\circ}\right)$ (see Fig. 2$)$. We then generate a noise normally distributed on the S2 observations. We choose different standard deviations for the astrometry and the spectroscopy: $\sigma_{a}=(10,30) \mu$ as and $\sigma_{V}=(1,10) \mathrm{km} / \mathrm{s}$. The aim is to fit the models $\mathrm{A}, \mathrm{B}$ and $\mathrm{C}$ to the noisy observations generated with model $\mathrm{D}$, and determine the minimal observation time above which the different models fail to fit the data considering the different accuracy pairs $\left(\sigma_{a}, \sigma_{V}\right)$.

Considering the different results presented Table. 1, we can see that model A fails to fit the observations above a few months for each accuracy pair $\left(\sigma_{a}, \sigma_{V}\right)$. Regarding now 

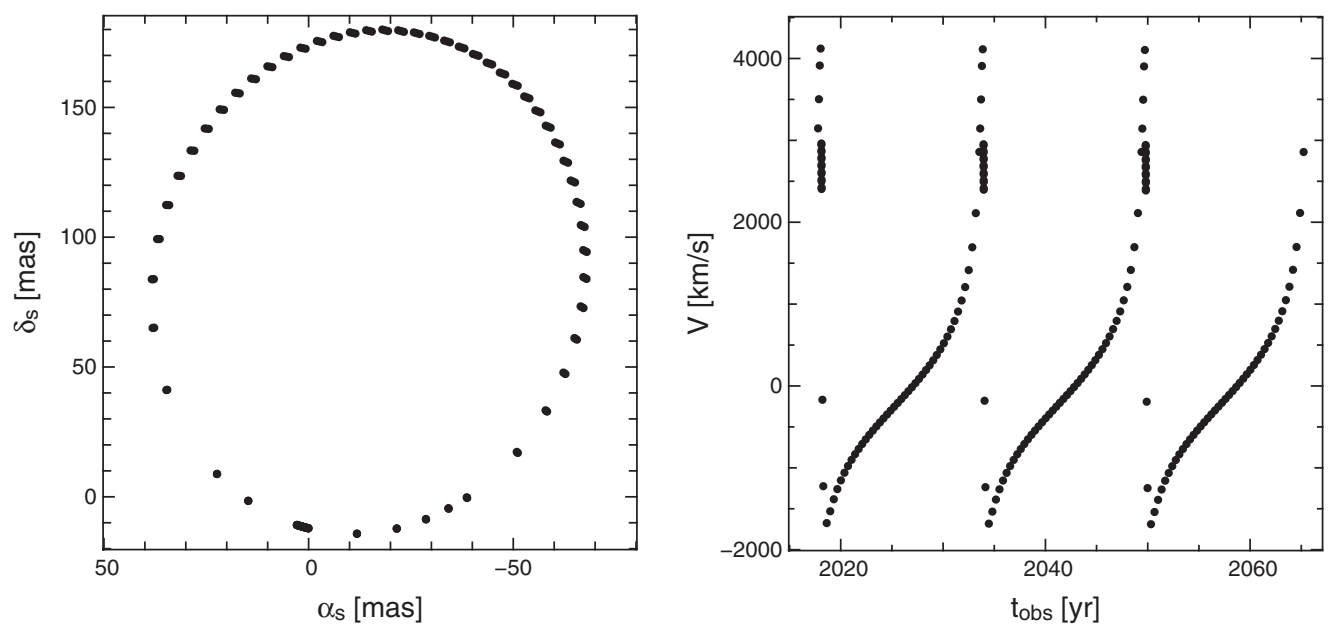

Figure 2. Astrometric (left) and spectroscopic (right) observations of the S2 star generated in full general relativity with model D. The observations start in 2017.7 and are obtained considering three S2 periods $(\approx 47$ years $)$. The observations are better sampled during pericenter passages.

model B, we note that the minimal observations times reached are higher. These differences are due to the fact that relativistic effects are included in model B but are missing in model A. They correspond to the transverse Doppler redshift and the gravitational redshift. Comparing models A and B for the different accuracy pairs considered here thus allows observers to detect these relativistic effects in a few months.

Focusing now on models $\mathrm{B}$ and $\mathrm{C}$ for $\left(\sigma_{a}, \sigma_{V}\right)=(10 \mu \mathrm{as}, 1-10 \mathrm{~km} / \mathrm{s})$, we note that the threshold times are similar for both models. It means that the models fail to reproduce the observations because of the same relativistic effects missing in both of them and correspond to the effects induced by the photon curvature. It is thus possible to be sensitive to the photon curvature by using models $\mathrm{B}$ and $\mathrm{C}$ for the accuracy pairs $\left(\sigma_{a}, \sigma_{V}\right)=(10 \mu \mathrm{as}$, $1-10 \mathrm{~km} / \mathrm{s})$. Regarding now the pairs $\left(\sigma_{a}, \sigma_{V}\right)=(30 \mu \mathrm{as}, 1-10 \mathrm{~km} / \mathrm{s})$, the threshold times obtained are different for both models. The differences between these models are the pericenter advance and the Lense-Thirring effect: they are included in model $\mathrm{C}$ but neglected in model B. However, the effect of the angular momentum of the black hole is negligible compared to the pericenter advance. For instance, at the first apocenter passage of the S2 star the Lense-Thirring effect reaches of about $15 \mu$ as and the pericenter advance reaches of about $500 \mu$ as (the spectroscopic impact of these two effects is negligible and is inferior to $3 \mathrm{~km} / \mathrm{s}$, see Fig 1 ). We can thus claim that the pericenter advance can be detected above typically one period of $\mathrm{S} 2$ considering $\left(\sigma_{a}, \sigma_{V}\right)=(30 \mu \mathrm{as}$, $1-10 \mathrm{~km} / \mathrm{s}$ ). This effect should be detected earlier if we consider a different model taking into account all relativistic effects such as the photon curvature but only neglecting the pericenter advance. Indeed, in such a case we will not be affected by the presence of high-order effects which is the case with model B for $\left(\sigma_{a}, \sigma_{V}\right)=(10 \mu \mathrm{as}, 1-10 \mathrm{~km} / \mathrm{s})$.

\section{Constraint on the angular momentum of the central black hole candidate}

We are also interested in the constraint on the norm and the direction of the compact source at the Galactic Center. To do so we use a model $\mathrm{C}+$ corresponding to an 


\begin{tabular}{|c|c|c|}
\hline Model A & $10 \mu$ as & $30 \mu$ as \\
\hline $1 \mathrm{~km} / \mathrm{s}$ & 1 month & 1 month \\
$10 \mathrm{~km} / \mathrm{s}$ & 2 months & 4 months \\
\hline Model B & $10 \mu$ as & $30 \mu$ as \\
\hline $1 \mathrm{~km} / \mathrm{s}$ & 8 months & 13 years \\
$10 \mathrm{~km} / \mathrm{s}$ & 6 years & 18 years \\
\hline Model C & $10 \mu$ as & $30 \mu$ as \\
\hline $1 \mathrm{~km} / \mathrm{s}$ & 6 months & $>30$ years \\
$10 \mathrm{~km} / \mathrm{s}$ & 6 years & $>30$ years \\
\hline
\end{tabular}

Table 1. Approximation of the minimal observations times above which the models A, B and $\mathrm{C}$ fail to fit the different S2 star noisy observations generated with the full general relativity model, D.

improvement of model $\mathrm{C}$ : it takes into account the same effects as model $\mathrm{C}$ but considers gravitational lensing through analytical approximations developed in weak deflection limit and weak field (Sereno et al. 2006). We fit model C+ to observations simulated with the full general relativity model, D. The aim is to determine whether we can detect the Lense-Thirring effect without considering ray-tracing. The observations are generated considering the same angular momentum parameters as previously: $\left(a, i^{\prime}, \Omega^{\prime}\right)=\left(0.99,45^{\circ}, 160^{\circ}\right)$.

If we consider observations of the $\mathrm{S} 2$ star obtained during three full orbital periods (from 2017.7 to 2065$)$ and an accuracy pair $\left(\sigma_{a}, \sigma_{V}\right)=(10 \mu \mathrm{as}, 10 \mathrm{~km} / \mathrm{s})$ we find that the spin is constrained with $1 \sigma$ uncertainty of about 0.1 . The angles $i^{\prime}$ and $\Omega^{\prime}$ are constrained with $1 \sigma$ uncertainty of about $14^{\circ}$ and $24^{\circ}$, respectively.

These results are consistent with those obtained by Yu et al. 2016 where the authors fit a model similar to our model D to astrometric and spectroscopic observations of the S2 star generated with this same model. In other words, the authors used a full general relativity model to constrain the different parameters. We show that even without using a ray-tracing code it is possible to converge to the same strong constraint on the angular momentum parameters as those obtained with a full general relativity model.

\section{Conclusion}

Combining the GRAVITY instrument and accurate spectrographs $\left(\sigma_{V}=1-10 \mathrm{~km} / \mathrm{s}\right)$ is expected to lead to detection of both low- and high-order relativistic effects. In particular, it is possible to put in light transverse Doppler and gravitational redshifts in a few months or to be sensitive to the photon curvature in several months considering spectroscopic accuracies of about $1 \mathrm{~km} / \mathrm{s}$. By using the models implemented here the pericenter advance can only be detected after approximatively one orbital period of the S2 star for an astrometric accuracy of about $30 \mu \mathrm{as}$. However, it should be detected earlier if we fit a model similar to model B but taking into account the photon curvature. Indeed, in such a case we will not be affected by high-order effects that cause model B to fail for $\left(\sigma_{a}, \sigma_{V}\right)=(10 \mu \mathrm{as}, 1-10 \mathrm{~km} / \mathrm{s})$. It should be also possible to detect the pericenter advance earlier if using another observation strategy such as considering previous observations of the S2 star in addition to the new data. The Lense-Thirring effect is also observable. Strong constraint on the norm and the direction of the angular momentum of the black hole can be obtained considering long monitoring of the S2 star (3 periods of S2). Reasonable constraints on these parameters should be reached even considering observations obtained during two periods of the S2 star. 
In order to improve the study performed here it is necessary to take into account the Newtonian effect induced by the presence of an extended mass at the center of our Galaxy (Rubilar et al. 2001, Weinberg et al. 2005). Even if this mass is not constrained by the observations it is important to determine whether the different effects that can be detected by GRAVITY with the S2 star are still constrained.

\section{References}

Angélil, R. and Saha, P., \& Merritt, D. 2010, ApJ, 720, 1303

Bozza, V. \& Mancini, L. 2012, ApJ, 753, 56

Broderick, A. E., Fish, V. L., Doeleman, S. S., \& Loeb, A. 2011, ApJ, 735, 110

Davies, R., Ageorges, N., Barl, L., Bedin, L. R., Bender, R., Bernardi, P., Chapron, F., Clenet, Y., Deep, A., Deul, E., Drost, M., Eisenhauer, F., Falomo, R., Fiorentino, G., Förster Schreiber, N. M., Gendron, E., Genzel, R., Gratadour, D., Greggio, L., Grupp, F., Held, E., Herbst, T., Hess, H.-J., Hubert, Z., Jahnke, K., Kuijken, K., Lutz, D., Magrin, D., Muschielok, B., Navarro, R., Noyola, E., Paumard, T., Piotto, G., Ragazzoni, R., Renzini, A., Rousset, G., Rix, H.-W., Saglia, R., Tacconi, L., Thiel, M., Tolstoy, E., Trippe, S., Tromp, N., Valentijn, E. A., Verdoes Kleijn, G., \& Wegner, M. 1995, in Proc. SPIE, Vol. 7735

Doeleman, S. S., Fish, V. L., Broderick, A. E., Loeb, A., \& Rogers, A. E. E. 2009, ApJ, 695, 59

Eisenhauer, F., Abuter, R., Bickert, K., Biancat-Marchet, F., Bonnet, H., Brynnel, J., Conzelmann, R. D., Delabre, B., Donaldson, R., Farinato, J., Fedrigo, E., Genzel, R., Hubin, N. N., Iserlohe, C., Kasper, M. E., Kissler-Patig, M., Monnet, G. J., Roehrle, C., Schreiber, J., Stroebele, S., Tecza, M., Thatte, N. A., \& Weisz, H. 2003, in Proc. SPIE, Vol. 4841

Fragile, P. C. \& Mathews, G. J. 2000, ApJ, 542, 328

Ghez, A. M., Salim, S., Weinberg, N. N., Lu, J. R., Do, T., Dunn, J. K., Matthews, K., Morris, M. R., Yelda, S., Becklin, E. E., Kremenek, T., Milosavljevic, M., \& Naiman, J. 2008, ApJ, 689,1044

Gillessen, S., Eisenhauer, F., Trippe, S., Alexander, T., Genzel, R., Martins, F., \& Ott, T. 2009, ApJ, 692, 1075

Jaroszynski, M. 1998, Acta Astron., 48, 653

Kannan, R. \& Saha, P. 2009, ApJ, 690, 1553

McLean, I. S., Becklin, E. E., Figer, D. F., Larson, S., Liu, T., \& Graham, J. 1995, in Proc. SPIE, Vol. 2475

Rubilar, G. F. \& Eckart, A. 2001, A\& A, 374, 95

Sereno, M. \& de Luca, F. 2006, Phys. Rev. D, 74, 123009

Vincent, F. H., Paumard, T., Gourgoulhon, E., \& Perrin, G. 2011, Classical and Quantum Gravity, 28, 225011

Vincent, F. H., Meliani, Z., Grandclément, P., Gourgoulhon, E., \& Straub, O. 2016, Classical and Quantum Gravity, 33, 105015

Weinberg, N. N., Milosavljević, M., \& Ghez, A. M. 2005, ApJ, 622, 878

Will, C. M. 2008, ApJ, 674, L25

Yu, Q., Zhang, F., \& Lu, Y. 2016, ArXiv e-prints

Zhang, F., Lu, Y., \& Yu, Q. 2015, ApJ, 809, 127

Zucker, S., Alexander, T., Gillessen, S., Eisenhauer, F., \& Genzel, R. 2006, ApJ, 639, L21 